\begin{tabular}{|c|c|}
\hline - cogninto & 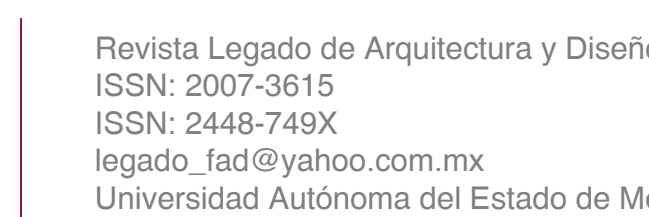 \\
\hline
\end{tabular}

\title{
EL DISEÑO BIOFÍLICO Y SU RELACIÓN CON EL MOBILIARIO URBANO
}

Escamilla-Cerón, Karla; Luna-Rodríguez, Sofía Alejandra

EL DISEÑO BIOFÍLICO Y SU RELACIÓN CON EL MOBILIARIO URBANO

Revista Legado de Arquitectura y Diseño, vol. 15, núm. 27, 2020

Universidad Autónoma del Estado de México, México

Disponible en: http://www.redalyc.org/articulo.oa?id=477963263011

Esta obra está bajo una Licencia Creative Commons Atribución-NoComercial-SinDerivar 4.0 Internacional. 


\title{
EL DISEÑO BIOFÍLICO Y SU RELACIÓN CON EL MOBILIARIO URBANO
}

\author{
BIOPHILIC DESIGN AND ITS RELATIONSHIP WITH \\ THE URBAN FURNITURE
}

\author{
Karla Escamilla-Cerón karla.escamillace@uanl.edu.mx \\ Universidad Autónoma de Nuevo León, México \\ Sofía Alejandra Luna-Rodríguez sofia.lunard@uanl.edu.mx \\ Universidad Autónoma de Nuevo León, México
} Revista Legado de Arquitectura y Diseño,
vol. 15, núm. 27, 2020

Universidad Autónoma del Estado de México, México

Recepción: 16 Agosto 2019 Aprobación: 24 Noviembre 2019

Redalyc: http://www.redalyc.org/ articulo.oa?id $=477963263011$
Resumen: Este proyecto surge a partir de la vinculación entre COSMOB (Italia) Laboratorio Pesaro (Italia), y la Licenciatura en Diseño Industrial, de la Facultad de Arquitectura en la Universidad Autónoma de Nuevo León (México), cuyo objetivo es desarrollar propuestas innovadoras de mobiliario público para exterior, con la aplicación de Monolite Ipergres, material cerámico patentado por dicha empresa y por la inquietud de diversificar el uso del mismo abordando otros entornos más allá de los tradicionales en los que se ha empleado comúnmente.

Dicho proyecto se trabajó con un enfoque centrado en el material, a través de una investigación de corte cualitativo, por medio de una encuesta exploratoria para la primera fase de la investigación, las cuales arrojaron el enfoque del tema que se aborda: mobiliario urbano enfocado centrado en la movilidad, con un enfoque sustentable, el cual se logra por la tipología de este material; así como entrevistas, análisis de información y observación para conocer y establecer propuestas más viables, las cuales se trabajaron por medio del diseño analógico a partir del diseño biofílico, término que etimológicamente significa "amor por la vida", pero es más que eso, en La hipótesis de la biofilia (1993), Kellert y Wilson se refieren a que la naturaleza tiene un vínculo directo con nuestro bienestar cognitivo, espiritual y emocional. Por lo que, Frattale toma como inspiración estos patrones y el tipo de diseño para brindar un estímulo más interesante y cambiante a las ciudades que contribuya al bienestar humano, todo esto por medio de mobiliario urbano.

Palabras clave: Diseño biofílico, espacios urbanos, fractal, mobiliario urbano.

Abstract: This project arises from a link between COSMOB (Italy) Laboratorio Pesaro (Italy), and the degree in Industrial Design, from the Faculty of Architecture at the Autonomous University of Nuevo León (Mexico), with the aim of developing innovative proposals for public outdoor furniture, with its main material "monolith Ipergress" ceramic material patented by said company and for the concern to diversify its use by addressing other environments beyond the traditional ones in which it has been commonly used.

This project was worked with a material-centered approach, through qualitative research, through an exploratory survey for the first phase of the research, which showed the focus of the topic being addressed: focused urban furniture focused on mobility, with a sustainable approach which is achieved by the typology of this material; as well as interviews, information analysis and observation to know and establish the most viable proposal, which were worked on through analog design based on biophilic design which is a term that etymologically means "love for life" but is more than that, in The Biophilia Hypothesis (1993) Kellert, Stephen and EO Wilson refer to the fact that nature has a direct link with our cognitive, spiritual and emotional well-being. Therefore, Frattale takes as inspiration these patterns and the type of design to provide a more interesting and changing stimulus to cities that contributes to well-being. human, all this by means of urban furniture.

Keywords: Biophilic design, urban spaces, fractal, urban furnishing. 


\section{INTRODUCCIÓN}

Actualmente, la tasa de urbanización en Nuevo León ha aumentado considerablemente, habitan más de 5 millones de personas y se ocupa el octavo lugar a nivel nacional por su número de habitantes, según datos del INEGI en 2015, debido a lo anterior, se observa cómo se empieza a crear una desconexión en el mundo natural por vivir dentro de la ciudad y la industria, esto se puede ver directamente en lo relacionado a la movilidad, que en términos de diseño es uno de los mayores desafíos a enfrentar en estos tiempos, la intensa urbanización de los territorios, el aumento del uso del automóvil, el crecimiento poblacional y el ritmo de vida de las personas crean un fenómeno de colapso en las conexiones establecidas o disparan parámetros en los nuevos proyectos. La intensidad y frecuencias de las actividades cotidianas en aumento determinan a su vez algunas de las características funcionales de la ciudad. Como menciona Rodríguez (2013), lo cual nos lleva a centrarnos en esta problemática por medio del diseño de mobiliario urbano, con enfoque biofílico.

En 1980, el diseño biofílico empezó a popularizarse por el biólogo estadounidense Edward O. Wilson, debido a la falta de contacto de las personas con la naturaleza, ya que dicho diseño tiene como principios crear un enfoque centrado en el ser humano, mejora los espacios donde habitan y trabajan las personas. Además, se genera una conexión con la naturaleza, lo que ayuda a beneficiar la salud al establecer contacto con el exterior, también contribuyen en la reducción del estrés y crean tranquilidad hacia la persona. $\mathrm{Al}$ implementar elementos naturales como luz solar, uso de maderas, patrones, tonalidades, entre otros, es una forma de aludir a la naturaleza y generar automáticamente un cambio en los espacios interiores y exteriores.

Como menciona Oliver Heath (2018), director de Oliver Heath Design, "el diseño biofílico debería considerarse una filosofía de diseño universal en lugar de una mera tendencia de diseño más". Por lo tanto, a través de este texto se explica el impacto del diseño biofílico y cómo fue utilizado para el desarrollo del proyecto.

\section{EL DISEÑO BIOFÍLICO Y SUS PRINCIPALES BENEFICIOS}

El diseño biofílico, desde tiempo atrás, se ha tomado como un fundamento de los beneficios buscados desde esa perspectiva del diseño al integrar el ambiente natural al artificial a favor del ser humano y brindando un enfoque para crear edificios y espacios que respondan a las necesidades humanas. Los principios de éste se pueden aplicar, tanto a la creación de objetos o edificios nuevos como existentes, así como espacios de interior y exterior, logrando elementos físicos que generan asociaciones entre los demás componentes del entorno, además de dar pie para lograr relaciones sociales que, como espacio público, se dan en su medio construido. 
Las relaciones de la sociedad en un espacio público generadas por elementos urbanos definen a grandes rasgos el fenómeno de la identidad urbana. Un ejemplo, es la creación de una plaza fundacional en los pueblos, ya que constituyen parte esencial del paisaje urbano teniendo al mobiliario como elemento conformador del espacio público, el cual se compone por elementos físicos, tanto artificiales como naturales, conformando el entorno cotidiano en coexistencia con los ciudadanos. Según Morgan Ball (2006), el ciudadano debe ser considerado como elemento importante del paisaje urbano y por esta razón las intervenciones en los espacios de confluencia social deben ser analizadas cuidadosamente para contribuir a las relaciones y a su permanencia como usuario.

Sin embargo, las personas cada vez pasan más tiempo en espacios cerrados y entornos urbanos, por lo que se buscan aumentar el contacto con la naturaleza. Como mencionan varios investigadores de Interfase del Design Lab, "un ambiente sin naturaleza puede tener un efecto negativo en la salud, productividad y bienestar". De tal manera que las personas suelen preferir vistas naturales en espacios urbanos, ya que provocan estados emocionales y psicológicos positivos (DesignLab, 2018). Además, al agregar referencias de la naturaleza en los diseños por medio de analogías, ya sea con materiales, patrones, texturas o colores, ayudan a que los individuos se sientan mejor, creando un efecto psicológico positivo.

Un ejemplo de esto es la obra "Ciudades jardín del mañana", en la cual Ebenezer Howard logró una obra fundamental del nuevo modelo de urbanismo de principios de siglo XX y que influiría posteriormente en el concepto de ciudad suburbana a partir de la década de los cuarenta (Montiel, 2015). Es un ejemplo de obras arquitectónicas que integraron la naturaleza en sus espacios, este modelo ha sido replicando en otras partes del mundo y sirvió como punto de partida para lo que conocemos actualmente como biodiseño.

Pasando a referente en objetos, se parte de trabajar con formas basadas en la naturaleza como lo son los fractales, un patrón geométrico complejo y repetitivo, por el cual nuestro sistema neurológico responde positivamente a través de su efecto regenerativo, según lo mencionado por el matemático Nikos Salingaros.

De acuerdo con "Building Biophilia: Connecting People to Nature in Building Design" incorporar estos elementos repetitivos en diseño de productos o en la arquitectura, estimula al cerebro humano llamándole a esta cualidad "diferencia dentro de la semejanza" (Hase y Heerwagen, 2000). Esto hace sentido, puesto que la gente piensa en espacios exteriores cuando se trata de apartar de la rutina o situaciones de estrés, lo cual actualmente lo vemos en varias manifestaciones como muros y azoteas verdes, donde se integra la arquitectura y los objetos con la naturaleza en beneficio de las personas. 


\section{METODOLOGÍA}

Se utilizó una metodología cualitativa, la cual se define como una investigación que produce datos descriptivos, ya que se consideran a las personas y el entorno en que les rodea, se observa el comportamiento, su panorama, además de realizarse investigación de campo. Como menciona Taylor y Bogdan (1989), en unos de sus criterios de la definición de metodología cualitativa: "los estudios cualitativos dan énfasis a la validez de la investigación, aseguran un estrecho ajuste entre los datos y lo que realmente la gente hace y dice. Observando a las personas en su vida cotidiana. Creando conceptos, definiciones y clasificaciones", lo cual aplicaba completamente a este proyecto, con alcance descriptivo, analítico y explicativo, ya que mediante estos se puede demostrar las relaciones entre los fenómenos que se estudian y llegar a las causas que originan estos.

Para esta investigación se utilizaron las herramientas de observación, entrevistas y análisis documental que al final brindaron datos para el beneficio de la investigación. Donde la observación cualitativa se utilizó para recabar información y datos específicos de lo que se estaba viendo, lo que se está sintiendo, como señala Patricia y Peter Adler, la observación consiste en obtener impresiones del mundo circundante por medio de todas las facultades humanas relevantes" (Álvarez-Gayou-Jurgenso et al., 2010).

En cuanto a la observación, se creó un diario de campo de las observaciones, recopilando información de las personas en espacios públicos y su movilidad, así como lo referente a las formas que nos brinda la naturaleza, además de analogías que al final contribuyeron con la propuesta final de diseño.

Así como se aplicaron entrevistas diagnósticas para conocer la percepción de las personas, al inicio de la investigación posteriormente complementándose con entrevistas conversacionales.

Con la ayuda de estas herramientas cualitativas, se llevaron a cabo cuatro fases en este proceso metodológico, las cuales dan como resultante establecer el resultado de la propuesta (figura 1). 


\section{/ METODOLOGÍA}

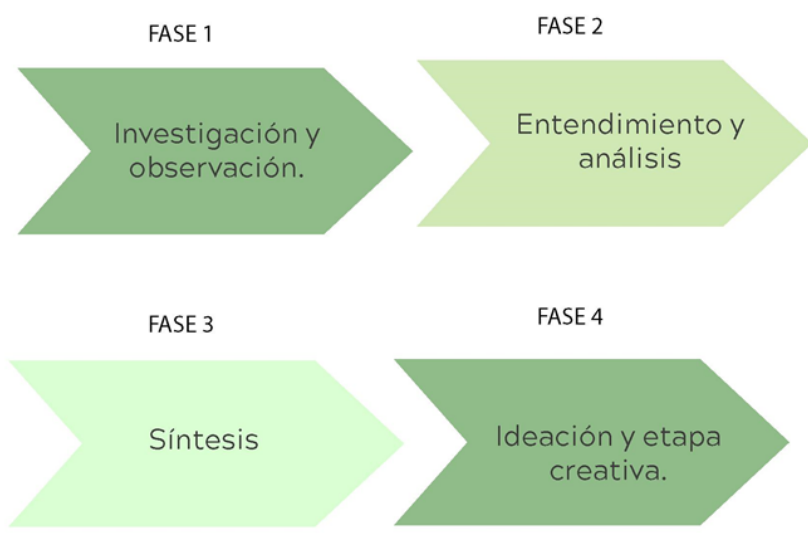

Figura 1. Esquema general metodológico aplicado. Fuente: Elaboración propia (2019).

A continuación, se describen de manera general cada uno de las fases del proyecto.

\section{Fase 1. Investigación y observación}

Se recoleta información de las variables: movilidad, mobiliario urbano, materiales cerámicos, entre otras, para conocer el contexto general y pasar a la observación de manera directa y específica, dándonos a la tarea de observar y entender los espacios exteriores donde se da la movilidad no motorizada, así como determinar qué elementos existían a su alrededor detectando patrones y elementos clave en los espacios.

\section{Fase 2. Entendimiento y Análisis}

Se analizaron las tendencias dentro de mobiliario como la referente a los materiales naturales, aspectos de sustentabilidad tan en boga actualmente, así como el minimalismo presente en las propuesta actuales de éste y la mega tendencias movilidad entendiendo las relaciones espaciales, dimensionales, de velocidad y de dirección, así como del usuario situándolo en dos escenarios con características físicas similares, están en la misma cuidad y área, pero a la vez diferentes, estableciendo un recorrido (como elemento representativo) por el primer cuadro de la cuidad (calles Zaragoza y Padre Mier), y otro siendo el recorrido por centro de la cuidad (avenida Juárez). El primer cuadro de la cuidad presenta amplios espacios naturales y mobiliario que apoya el descanso y en la avenida Juárez, las dimensiones de los recorridos son menores, el tránsito es más concurrido y el ritmo más acelerado. Como es lógico 
suponer los parámetros mencionados serían distintos en ambos escenarios y es precisamente la influencia del mobiliario urbano, quien determina la movilidad en este caso, el cual determinó al llegar a algunos insights que establecieron el rumbo del proyecto.

En el aspecto formal se utilizó como referente la geometría fractal, para establecer lo referente a esto en relación con la percepción del usuario, se llevaron a cabo una serie de preguntas ligadas a la movilidad y los problemas que a su consideración serían los más importantes a resolver, además de conocer su opinión sobre el mobiliario urbano y qué efecto positivo o negativo tenía dentro de sus actividades.

\section{Fase 3. Sintesis}

Con la información recabada se logró llegar a una serie de particularidades, con el cual se trabajó bajo la herramienta de insights combination, así como la creación de una persona, para guiar el perfil de usuario con las cuales hacer una combinación entre tendencias y preferencias del usuario, donde el equipamiento del espacio y su diseño comienzan a jugar un papel fundamental. Algunos autores han llevado a cabo investigaciones demostrando como un mal diseño o carencia de mobiliario urbano provoca disminución de confluencia de personas en estos espacios y por consiguiente de las interacciones sociales (Ortiz, 2014).

Los elementos urbanos que conforman a la ciudad se encuentran en constante interacción, tanto entre ellos como con los seres humanos. Es esta interacción entre entorno y ser humano, definida principalmente como intercambio de información, las que se destacan en la explicación de los fenómenos en las ciudades y su relación con los objetos que la conforman, y que llega hasta la forma, la función y el material que conforma dichos objetos.

\section{Fase 4. Ideación y etapa creativa}

Parte de un análisis a raíz de la realización de una exploración de formas que resolviera de manera funcional, sustentable y estética diversos elementos de mobiliario para el exterior, con aporte de innovación y diseño, con las consideraciones de fabricación que el material establece por ser un material cerámico y poco explorado en la tipología de los objetos propuestos. 


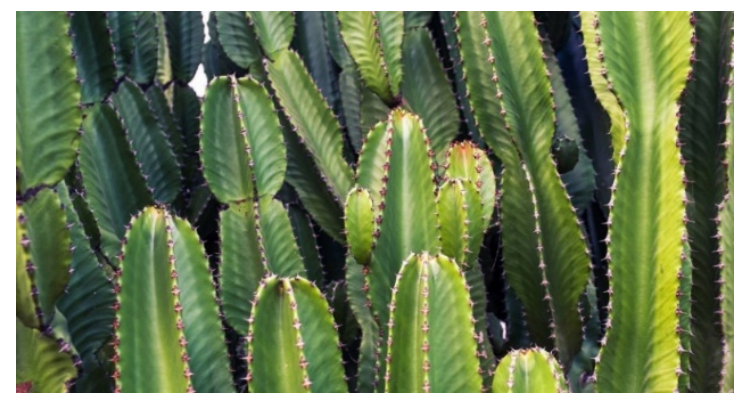

Figura 2. Base para analogía formal.

Fuente: Cactáceas (2016). Disponible en https://ecoosfera.com/2016/02/

por-que-los-cactus-tienen-espinas-en-lugar-de-hojas/.

Dichas fases vienen a responder al objetivo general de la investigación, como ya se mencionó, fue el diseño de un mobiliario urbano para exterior que apoyara la movilidad, con enfoque sustentable a través del material Monolite Ipergres.

Y cuyos objetivos específicos fueron, el generar elementos para un espacio al aire libre que promoviera la movilidad no motorizada y con esto la activación en espacios públicos, además de generar una experiencia positiva y de bienestar, incorporando elementos alineados a la naturaleza, aprovechar las características cerámicas del material para el desarrollo del elemento exterior; lo que da origen a frattale.

Frattale nace de evocar a la naturaleza a través del diseño biofílico. La inspiración principal son los patrones geométricos encontrados en la naturaleza llamados fractales, los cuales tienen un efecto positivo y de bienestar en nuestras mentes. Las formas resultantes fueron el producto de la exploración de los fractales encontrados en las cactáceas (figura 3) y la evolución de las mismas (figura 4).
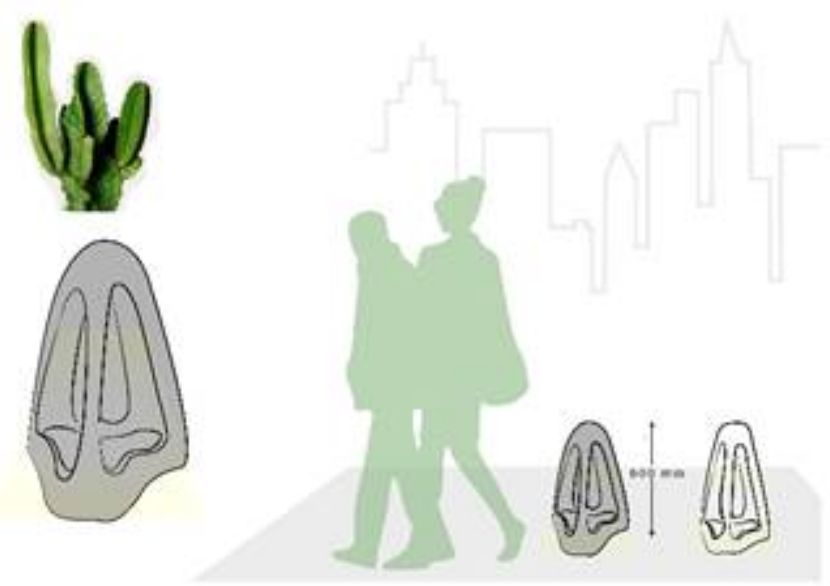

Figura 3. Analogía de cactáceas para rack de bicicletas. Fuente: Elaboración propia (2019). 


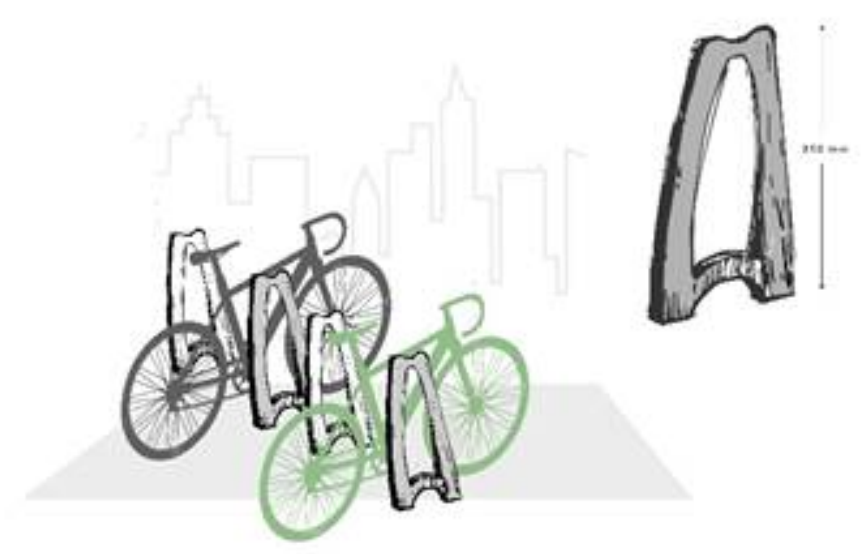

Figura 4. Evolución formal, rack para bicicletas. Fuente: Elaboración propia.

La propuesta incluye el desarrollo de un rack para bicicletas (figura 5), que podrán ser implementadas en espacios públicos o privados. El diseño y la simplicidad de las formas en cada componente, hace que las modulaciones sean diversas e interesantes esperando lograr en el usuario una experiencia diferente, la cual genera establecer un vínculo entre el usuario y el objeto, por el aspecto formal relacionada a la naturaleza, así como a la sencillez en su funcionalidad. Las propuestas activan el uso de espacios públicos y dan un sentido de pertenencia e identidad. El material propuesto es "Monolite Ipergres", parte clave de la vinculación y patentado por Laboratorio Pesaro como un material cerámico sustentable, que gracias a la mezcla de sus componentes y tecnología aseguran calidad y alta resistencia a exteriores, el cual cuenta con una utilización más tradicional como sería en bajillas, jarrones, artículos de decoración, entre otros. Lo que presentaba un reto para este proyecto debido a la tipología del producto, ya que los referentes a la aplicación de este tipo de material con que se contaba en el momento eran escasos. 


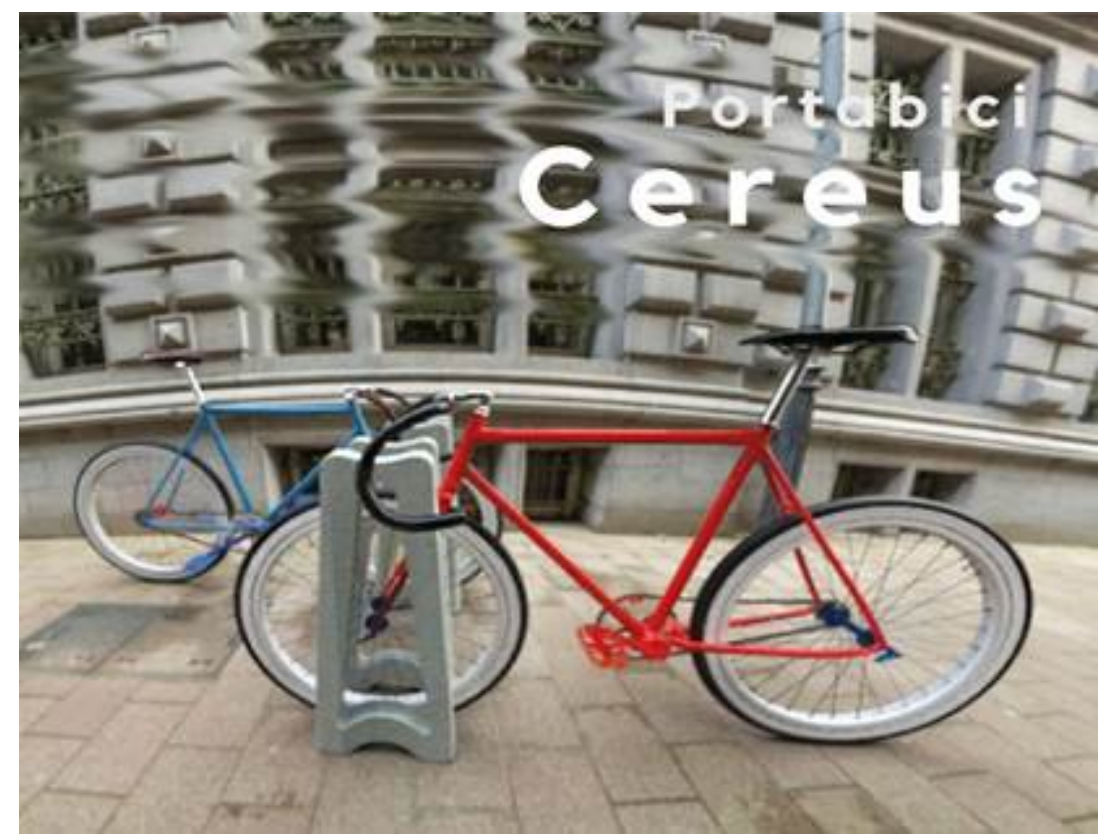

Figura 5. Propuesta final: Portabici Cereus. Fuente: Elaboración propia (2019).

Portabici Cereus es un sistema de módulos que resuelve de manera funcional, estética y sostenible el estacionamiento y aparcamiento de bicicletas. Logran cubrir el ámbito funcional además del estético, gracias al trabajo con analogías de la naturaleza que brida una forma agradable para la vista, lo cual desemboca en una óptima precepción del objeto, además la aplicación de color neutro, lo cual trasmite una sensación de limpieza formal y adaptable a una gran variedad de entornos; así mismo por la resistencia del material aunado de sus aspectos sostenible genera en suma un producto con aporte de diseño (figura 6).

Por lo tanto, este diseño contribuye a entornos urbanos que complementan la naturaleza, asimismo, son piezas conformadas con propiedades resistentes para el exterior. Es un objeto con base en formas geométricas y partiendo de la repetición creando una complejidad visual que el ojo del usuario acepta automáticamente, ya que los usuarios de un sistema urbano encuentran placentero el hecho de identificar patrones dentro de la complejidad mediante la percepción.

Formado por dos estructuras iguales, lo cual facilita su fabricación por requerir el diseño de un sólo molde para esto, así como un elemento central de unión. 


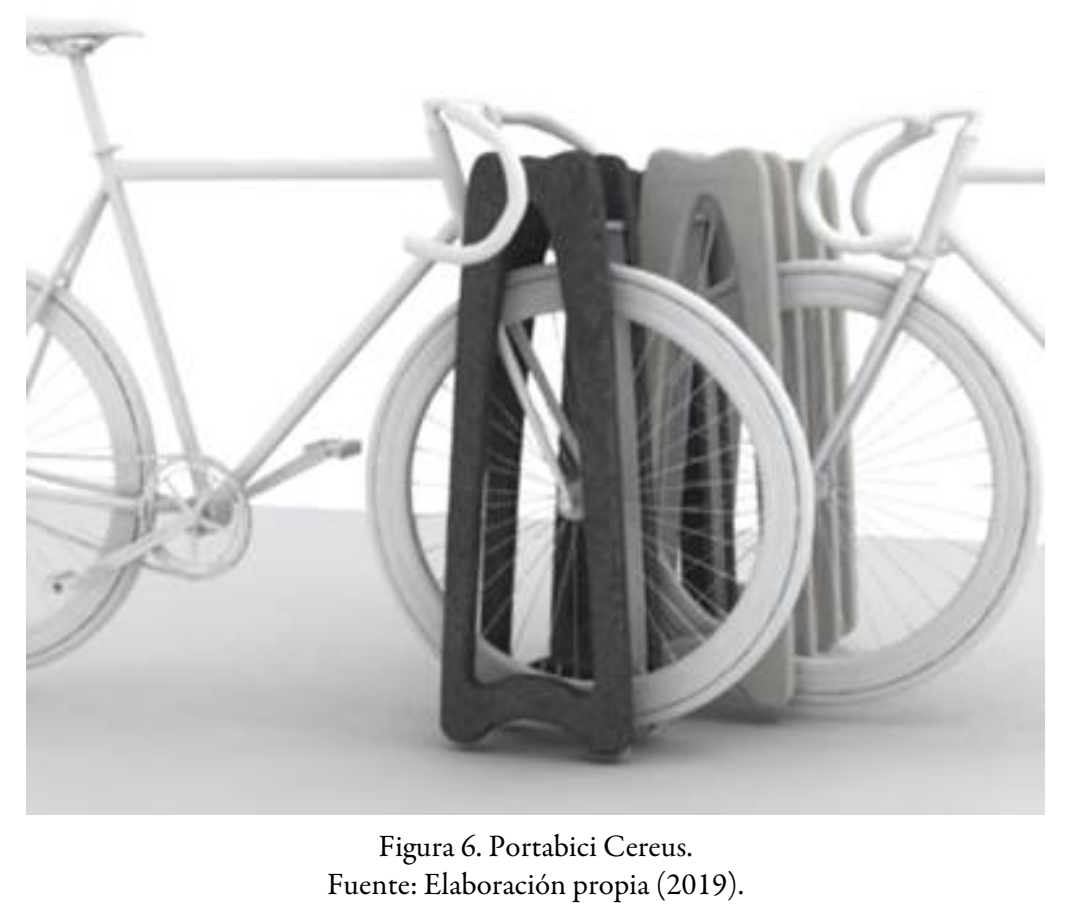

CONCLUSIONES

El uso del diseño basado en la naturaleza no significa implementar más árboles y plantas dentro de un espacio, sino de hacer posible las relaciones entre los elementos naturales y humanos en un entorno construido. Por medio del diseño e investigación se puede activar un espacio público reconfigurar un espacio y darle sentido de identidad y pertenencia. A partir de la investigación y desarrollo del proyecto se puede concluir que el hecho de que el diseño de mobiliario urbano en las ciudades no debe girar en torno al ser humano únicamente, como suele suceder con el diseño de elementos aislados. Este debe considerar interacciones entre actores y factores en todos los niveles que la componen y tener un factor guía como en este caso lo fue la implementación del material en este tipo de espacios con alusión formal a aspectos naturales en entornos urbanos para lograr generar en la mayoría de los casos impacto positivo en la percepción y por ende en el bienestar de las personas. Por lo que Frattale al tomar como inspiración estos patrones biofílicos logra conjugar los elementos para brindar un estímulo más interesante y cambiante a las ciudades que contribuya al bienestar humano, todo esto por medio de mobiliario urbano, ya que mediante la percepción que un ser humano tiene de un entorno se generan interpretaciones que permiten interrelacionar elementos o estímulos otorgando determinada estética a un espacio urbano. 


\section{FUENTES DE CONSULTA}

Álvarez-Gayou-Jurgenson, J. L., Camacho López, S. M., Martínez Campos, J. F., Solano Solano, G., Rodríguez Segura, E., López Ugalde, J. A. (2017), "Métodos básicos en la investigación cualitativa, la observación". BOLETÍN CIENTÍFICO "XIKUA", vol. 5, núm. 10. Universidad Autónoma del Estado de Hidalgo. Disponible en https://www.uaeh.edu.mx/scige/boletin/tlahuelilpan/ n10/r1.html\#: :text=La\%20observaci\%C3\%B3n\%20no\%20implica $\% 20 \%$ C3\%BAnicamente,todas\%20las\%20facultades\%20humanas \%20relevantes\%E2\%80\%9D, consultado el 24 de julio de 2018.

Hase, B., Heerwagen J. (2000), "Building Biophilia: Connecting People to Nature in Building Design" (Construyendo biofilia: conectando a las personas con la naturaleza en el diseño de edificios), Environmental Design \& Construction.

Interface (2019), Una introducción al Diseño Biofílico. Interface. Disponible en www.interface.com/LA/es-MX/campaign/biophilicdesign/Biophilic-Design-es_LA, consultado el 27 de septiembre de 2019.

Kellert, S. R., Wilson, O. E. (1993), "Biophilia and the conservation Ethic". En The Biophilia Hypothesis (31-35). Washington, DC: Island Press.

Montiel, T. (2015), Ebenezer Howard y La Ciudad Jardín (118). Madrid, ArtyHum. Disponible en https://www.aacademica.org/ teresa.montiel.alvarez/15.pdf, consultado el 2 de agosto de 2019.

Morgan Ball, D. (2006), "Los usuarios del espacio público como protagonistas en el paisaje urbano". Revista de Arquitectura (34-41). Disponible en http://editorial.ucatolica.edu.co/ojsucatolica/ revistas_ucatolica/index.php/RevArq/article/view/807/837, consultado el 14 de septiembre de 2019.

Oliver Heath Design (2018), Global Experts in Biophilic Design \& its Benefits to Health \& Well Being. Oliver Heath Design Ltd. Brighton. Disponible en https://www.oliverheath.com/, consultado el 21 de septiembre de 2018.

Ortiz López, A. E. (2014), Características que generan el éxito de los espacios públicos en Monterrey, Nuevo León. Tesis, Universidad Autónoma de Nuevo León, Monterrey.

Rodríguez Quintanilla, I. A. (2013), Percepción del usuario del transporte público con respecto a la calidad y el nivel de servicio ofertado, en las zonas metropolitanas de Aix-en Provence, Francia y Monterrey, México. Tesis, Universidad Autónoma de Nuevo León, Monterrey.

Taylor, S. y Bogdan R. C. (1989), Introducción a los métodos cualitativos de investigación, Paidós, Barcelona. 